\title{
STACKING SEQUENCE OPTIMIZATION OF COMPOSITE BEAMS WITH DIFFERENT LAYER THICKNESSES
}

\author{
Fatih Karaçam ${ }^{1}$, Taner Timarci ${ }^{1}$ \\ 1 Department of Mechanical Engineering, Faculty of Engineering, Trakya University, Edirne 22030, Turkey, \\ e-mail: fatihkar@trakya.edu.tr; tanert@trakya.edu.tr
}

Received: 2015.04.02

Accepted: 2015.05.08

Published: 2015.06.01

\begin{abstract}
In this study, stacking sequence optimization of composite beams with different layer thicknesses is investigated for various boundary conditions. A unified shear deformation theory is used for analytical solution. The optimization process is carried out in order to obtain the minimum deflection parameters for Clamped-Free (C-F), Clamped-Clamped (C-C) and simply supported (S-S) boundary conditions under a uniform distributed load by use of genetic algorithm for a specific number of population and generation. Finally, among all possible combinations of layer thicknesses, the one giving the minimum deflection parameter and corresponding stacking sequence is chosen. The minimum values and corresponding stacking sequences are presented for different boundary conditions.
\end{abstract}

Keywords: laminated composite beams, static analysis, genetic algorithm, layer thickness, stacking sequence optimization.

\section{INTRODUCTION}

Composite materials have gained popularity in modern structures where light-weight and high strength are sufficient to carry various loading types for different boundary conditions. Because of high elasticity modulus and strength, composite materials are more popular than any conventional materials, besides, the advantage of being designed with many parameters such as stacking sequence, layer thicknesses, material and geometrical properties, structures can be designed effectively in order to satisfy the desired mechanical properties. Walker and Smith [1] studied the ending analysis of laminated composite plates by minimizing a certain fitness function with genetic algorithm (GA). Due to the manufacturing constraints, they chose the sets of values from the ply angles and layer thicknesses. Conti et al. [2] described a method to optimize the thickness balance within a composite laminate with layers oriented according to a limited set of angles and used a finite element method in optimization process. Lee et al. [3], used a parallel evolutionary algorithm in multi-objective optimization of the stacking sequences in composite plates by choosing fiber type, thickness and orientation angles as design parameters for each layer. Brighenti [4], investigated the optimum fiber orientation of fiber reinforced composite materials in order to optimize a certain fitness function by use of GA. Khosravi and Sedaghati [5], considering the thickness of layers and fiber orientation angles as the design variables, presented two optimality criteria for optimum design of composite laminates using a finite element method. In the study, stacking sequence optimization of composite beams with different layer thicknesses is investigated for various boundary conditions. In the static analysis, a unified shear deformation beam theory is used which was first applied to composite shells by Soldatos and Timarc1 [6]. Initially, the layer thicknesses are generated so as to satisfy the total thickness of a four-layered composite beam. After the layer thicknesses are set, a random initial population of stacking sequences are used and evolved in each generation in order to obtain the minimum deflection parameter. Finally, among all the possible 
combinations of layer thicknesses, the one that has the minimum deflection parameter and corresponding stacking sequence is presented.

\section{ANALYTICAL METHOD}

In the study, the beam is assumed to be constructed of four linearly elastic layers. Various boundary conditions are applied to the beam under the effect of a uniform distributed transverse load. The beam has a unith width, length of $L$ and thickness of $h$ in a typical coordinate system where $0 \leq x$ $\leq L$ and $-h / 2 \leq z \leq h / 2$ as shown in Figure 1.

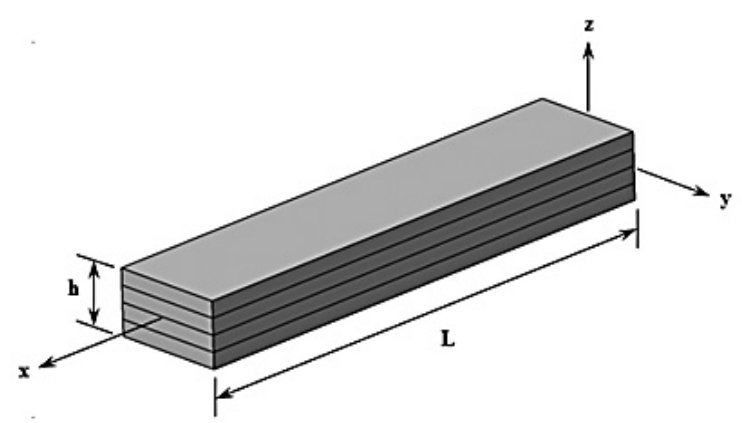

Fig. 1. Beam geometry and coordinate system

Uniform shear deformation beam theory is used in the static analysis where the other beam theories can be obtained as a special case [6]. The displacement fields are given by:

$$
\begin{gathered}
U(x, z)=u(x)-z w(x)_{, x}+\emptyset(z) u_{1}(x) \\
W(x, z)=w(x)
\end{gathered}
$$

where: $U, W$ are the displacement fields of the beam;

$u, u_{1}, w$ are the displacement fields of the mid-plane;

$\varnothing(z)$ represents the shape function that varies depending on the beam theory chosen [7].

By the use of the stress-strain relations into force $\left(N_{x}\right)$ and moment definitions $\left(M_{x}, M_{x}^{a}\right)$, the constitutive equations are given as below:

$$
\begin{gathered}
{\left[\begin{array}{l}
N_{x} \\
M_{x} \\
M_{x}^{a}
\end{array}\right]=\left[\begin{array}{ccc}
A_{11} & B_{11} & B_{111} \\
B_{11} & D_{11} & D_{111} \\
B_{111} & D_{111} & D_{1111}
\end{array}\right]\left[\begin{array}{c}
u_{, x} \\
-w_{, x x} \\
u_{1, x}
\end{array}\right],} \\
Q_{x}^{a}=A_{55} u_{1}
\end{gathered}
$$

where: $A_{i j}, B_{i j}$ and $D_{i j}$ denote the extensional, coupling and bending rigidities and $Q_{x}^{a}$ denotes the shear force respectively.
The rigidities with more than two indices and the moment with superscript correspond to the shear deformation beam theory and subscript comma indicates the derivation with respect to the relevant axis. The governing equations of composite beam for static analysis under the effect of a uniform distributed load are given as follows:

$$
\begin{gathered}
N_{x, x}=0 \\
M_{x, x x}=q(x) \\
M_{x, x}^{a}-Q_{x}^{a}=0
\end{gathered}
$$

The boundary conditions are prescribed at the edges of the beam when $x=0$ and $L$ are given for simply supported, cantilever and free cases respectively as follows:

$$
\begin{gathered}
N_{x, x}=w=M_{x}=M_{x}^{a}=0 \\
u=w=w_{, x}=u_{1}=0 \\
N_{x}=M_{x, x}=M_{x}=M_{x}^{a}=0
\end{gathered}
$$

The use of constitutive equations into governing equations, three unknown displacement fields of the mid-plane with eight unknown integration coefficients $\left(C_{k}\right)$ are obtained by integrating and solving the equations simultaneously as follows [8]:

$$
\begin{gathered}
u_{1}(x)=C_{1} e^{-p x}+C_{2} e^{p x}-\left(q x+C_{3}\right) \frac{D_{111}}{A_{55} D_{11}} \\
u(x)=-\frac{B_{111}}{A_{11}} u_{1}+C_{7} x+C_{8} \\
w(x)=\frac{D_{111}}{p D_{11}}\left[-C_{1} e^{-p x}+C_{2} e^{p x}-\right. \\
\left.-\frac{p}{A_{55}}\left(\frac{q x^{2}}{2}+C_{3} x\right)\right]+\frac{1}{D_{11}}\left(\frac{q x^{4}}{24}+C_{3} \frac{x^{3}}{6}\right) \\
+C_{4} \frac{x^{2}}{2}+C_{5} x+C_{6}
\end{gathered}
$$

where:

$$
p=\sqrt{\frac{-A_{55} A_{11} D_{11}}{D_{111}^{2} A_{11}-D_{1111} A_{11} D_{11}}}
$$

By use of a computational programme, the integration coefficients can easily be obtained by applying the boundary conditions at both edges of the beam.

\section{OPTIMIZATION METHOD}

Considering too many design parameters in an optimization problem will bring about too 
many solutions attendantly. Thus, instead of using all the possible solutions and obtain the optimum, using an evolutionary technique in the optimization process would be inevitable. Genetic algorithm is widely used in the design of laminated composite structures that have many variables and different fitness functions. Solving complex optimization problems by the use of conventional optimization techniques can be difficult in many fields. Genetic algorithm (GA) is an evolutionary optimization technique using Darwin's principal of survival of the fittest to improve a population of solutions [9]. The algorithm is generally based on natural selection, crossover and mutation which are also called genetic operators. Stacking sequences are the design variables that consist of fibre orientation angles $\left(\theta^{(k)}\right)$ where $k$ represents the number of layer. Therefore, the optimization problem may be written in a standard form such as:

Find: $\left[\theta^{(1)} / \theta^{(2)} / \theta^{(3)} / \theta^{(4)}\right]$

Minimize: $w$

Subject to: $0^{\circ} \leq \theta \leq 90^{\circ}$

The problem considered in the study is to optimize the stacking sequences of laminated composite beam in order to obtain the minimum deflection parameter among all possible combinations of layer thicknesses for various boundary conditions. In Figure 2, the cross-section of composite beam with the same and various layer thicknesses satisfying the total thickness of are presented respectively.

The flowchart of genetic algorithm used in the study is illustrated in Figure 3. After an initial population of stacking sequences is generated, they are ranked from minimum to maximum with corresponding deflection parameters. New individuals representing the stacking sequences are generated by crossover so as to swap one or two fiber orientation angle of two consecutive stacking sequences and the genetic diversity from one generation to another is maintained by mutation,

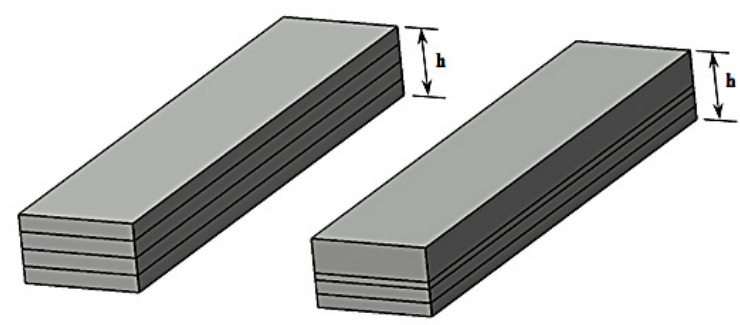

Fig. 2. Cross-section of the beam for the same and various layer thicknesses

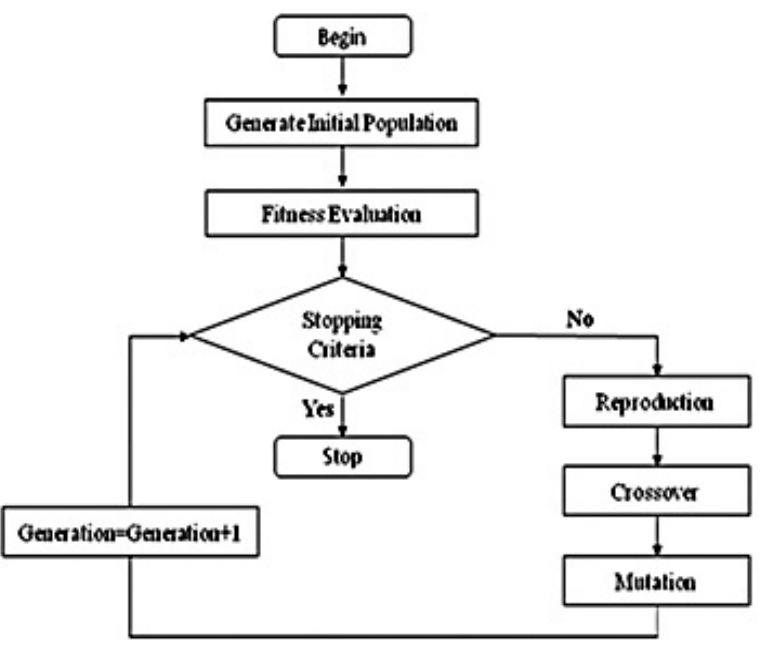

Fig. 3. The flowchart of genetic algorithm

altering one random fiber orientation angle of a random stacking sequence in each generation.

\section{RESULTS AND DISCUSSION}

The composite beam is assumed to be constructed of graphite/epoxy and under a uniform distributed load where $q(x)=1000 \mathrm{~N} / \mathrm{m}$, a length of $L$ $=1 \mathrm{~m}$ and thickness of $h=0,02 \mathrm{~m}$ and the mechanical properties of graphite/epoxy are given as [10]:

$$
\begin{gathered}
E_{11}=241.5 \mathrm{GPa}, E_{22}=E_{33}=18.89 \mathrm{GPa} \\
G_{23}=3.45 \mathrm{GPa}, G_{12}=G_{13}=5.18 \mathrm{GPa} \\
v_{23}=0.25, v_{12}=v_{13}=0.24
\end{gathered}
$$

In Table 1, the minimum deflection parameters and corresponding stacking sequences are presented for various layer thicknesses and boundary conditions. The first term in layer thicknesses and stacking sequences indicates the first layer at the bottom of the beam where the last indicates the one at the top. There may be too many possible combinations of layer thicknesses for a composite beam depending on the variation and the number of layers. For instance, in a four layered beam, although there is only one combination for a variation of $0.25 h$, there are 84 possible combinations for a variation of $0.1 \mathrm{~h}$. Since there are more possible combinations of layer thicknesses with a variation of $0.1 h$, the minimum deflection parameters are obtained for this case in all boundary conditions.

In Figure 4, Figure 5 and Figure 6, the variation of deflection parameters with respect to the number of generation for C-C, C-F and S-S boundary conditions are presented respectively. The algorithm is carried out for a population of 100 individuals and a number of generation of 50 in 
Table 1. The minimum deflection parameters and corresponding stacking sequences for various layer thicknesses and boundary conditions

\begin{tabular}{|c|c|c|c|c|}
\hline Boundary condition & Variation & Layer thicknesses $(\times h)$ & Deflection parameter [m] & Stacking sequence \\
\hline \multirow{3}{*}{ C-C } & 0.25 & $0.25 / 0.25 / 0.25 / 0.25$ & $1.75226 \cdot 10^{-5}$ & $0^{\circ} / 0^{\circ} / 0^{\circ} / 0^{\circ}$ \\
\cline { 2 - 5 } & 0.2 & $0.2 / 0.2 / 0.2 / 0.4$ & $1.75226 \cdot 10^{-5}$ & $0^{\circ} / 0^{\circ} / 0^{\circ} / 0^{\circ}$ \\
\cline { 2 - 5 } & 0.1 & $0.1 / 0.1 / 0.2 / 0.6$ & $1.10796 \cdot 10^{-6}$ & $0^{\circ} / 0^{\circ} / 0^{\circ} / 40^{\circ}$ \\
\hline \multirow{3}{*}{ C-F } & 0.25 & $0.25 / 0.25 / 0.25 / 0.25$ & $3.87065 \cdot 10^{-4}$ & $0^{\circ} / 10^{\circ} / 50^{\circ} / 40^{\circ}$ \\
\cline { 2 - 5 } & 0.2 & $0.2 / 0.2 / 0.2 / 0.4$ & $3.19941 \cdot 10^{-4}$ & $20^{\circ} / 90^{\circ} / 70^{\circ} / 0^{\circ}$ \\
\cline { 2 - 5 } & 0.1 & $0.4 / 0.1 / 0.4 / 0.1$ & $2.89024 \cdot 10^{-4}$ & $0^{\circ} / 10^{\circ} / 80^{\circ} / 0^{\circ}$ \\
\hline \multirow{3}{*}{ S-S } & 0.25 & $0.25 / 0.25 / 0.25 / 0.25$ & $8.22771 \cdot 10^{-5}$ & $0^{\circ} / 0^{\circ} / 10^{\circ} / 0^{\circ}$ \\
\cline { 2 - 5 } & 0.2 & $0.2 / 0.2 / 0.2 / 0.4$ & $8.24556 \cdot 10^{-5}$ & $0^{\circ} / 0^{\circ} / 10^{\circ} / 0^{\circ}$ \\
\cline { 2 - 5 } & 0.1 & $0.2 / 0.2 / 0.1 / 0.5$ & $8.19918 \cdot 10^{-5}$ & $0^{\circ} / 0^{\circ} / 10^{\circ} / 0^{\circ}$ \\
\hline
\end{tabular}

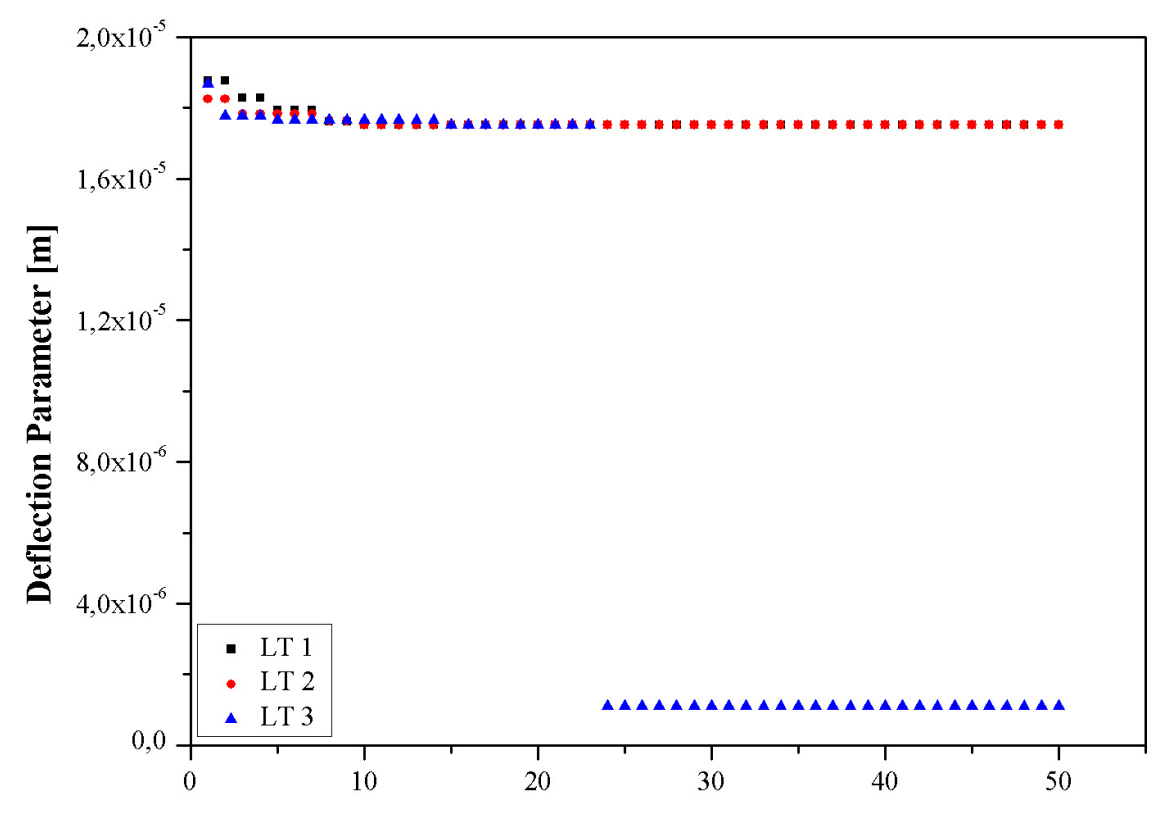

Number of Generation

Fig. 4. The variation of deflection parameters with respect to generation for $\mathrm{C}-\mathrm{C}$

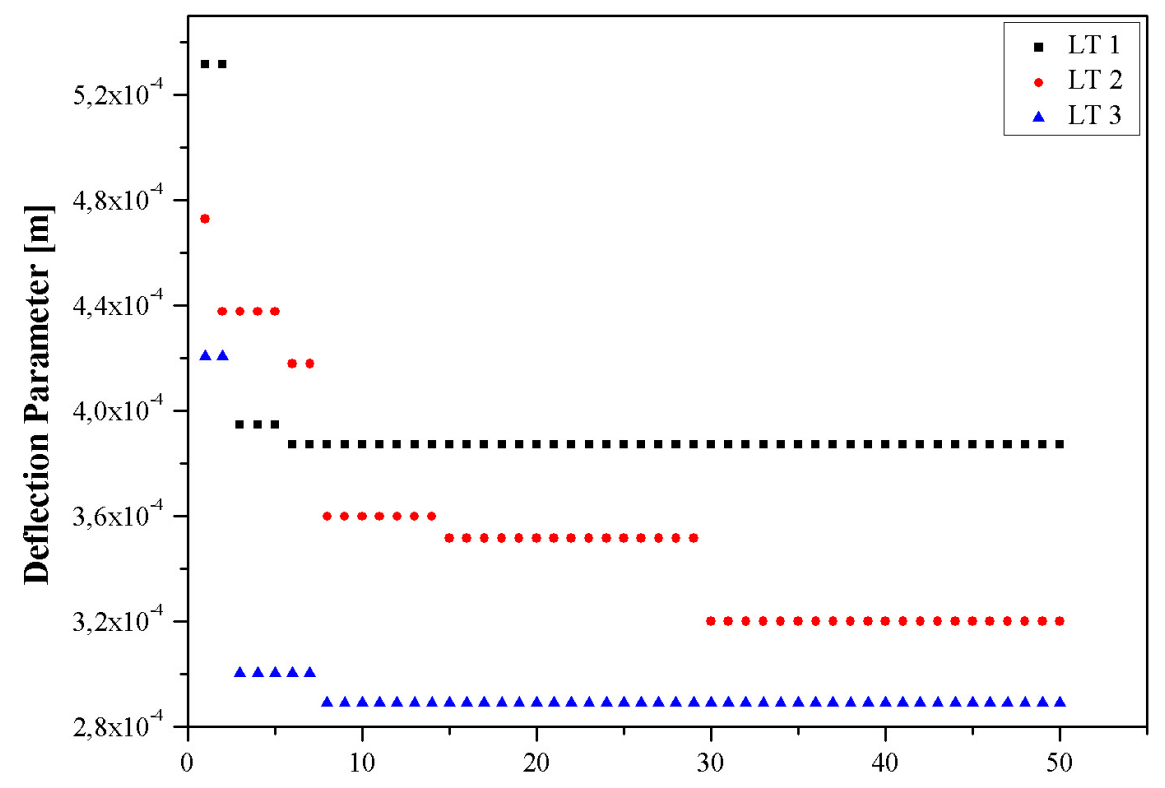

Number of Generation

Fig. 5. The variation of deflection parameters with respect to generation for C-F 


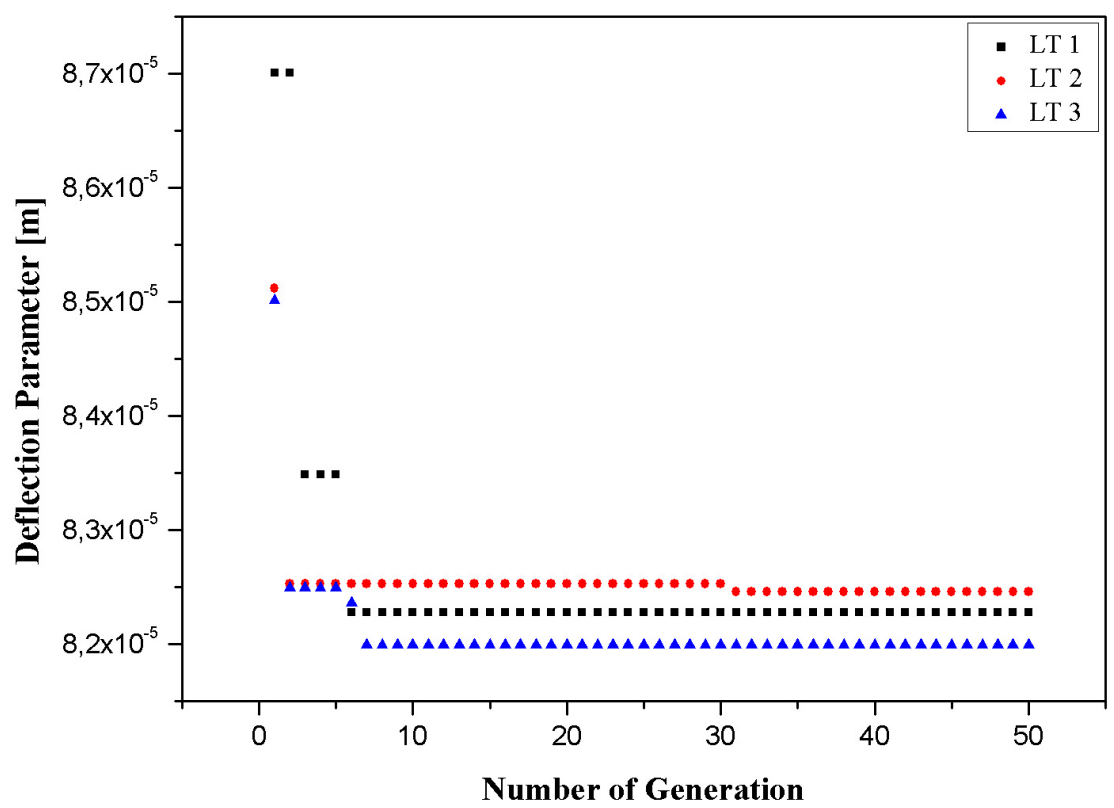

Fig. 6. The variation of deflection parameters with respect to generation for S-S

order not to stuck in a local minimum. The combination of layer thicknesses ( $L T)$, satisfying the total thickness of the beam together, are considered for three cases. In $L T 1$, the layer thicknesses are obtained for a variation of 0.25 which correspond to four layers with a same thickness of $0.25 \mathrm{~h}$. In $L T 2$ and LT3 the variation of 0.2 and 0.1 are considered respectively. As it is obvious from the figures that the minimum values are obtained for a variation of $0.1(L T 3)$ in all cases. For C-C boundary condition, the deflection parameters are very close to each other but the minimum value is obtained in $24^{\text {th }}$ generation. For C-F, the minimum value is obtained in $8^{\text {th }}$ generation and it is obtained in $7^{\text {th }}$ generation for S-S boundary condition.

\section{CONCLUSIONS}

In this study, the stacking sequence of a composite beam is optimized by the use of an evolutionary technique in order to obtain the minimum deflection parameters for various boundary conditions. Unlike the studies with the same layer thicknesses in the literature, the optimization process is carried out with different layer thicknesses. It is obvious from the results that, rather than the layers with the same thicknesses, the minimum values are obtained for different layer thicknesses due to the further number of possible combinations. In further works, the optimization problem can be expanded by taking new design parameters into account including strength, frequency and buckling.

\section{REFERENCES}

1. Walker M., Smith R.E.: A technique for the multiobjective optimisation of laminated composite structures using genetic algorithms and finite element analysis. Composite Structures, 62, 1, 2003, 123-128.

2. Conti P., Luparello S., Pasta A.: Layer thickness optimisation in a laminated composite. Composites Part B, 28B, 1997, 309-317.

3. Lee D.S., Morillo C., Bugeda G., Oller S., Onate E.: Multilayered composite structure design optimisation using distributed/parallel multi-objective evolutionary algorithms. Composite Structures, 94, 3, 2012, 1087-1096.

4. Brighenti R.: Fibre distribution optimisation in fibrereinforced composites by a genetic algorithm. Composite Structures, 71, 2005, 1-15.

5. Khosravi P., Sedaghati R.: Design of laminated composite structures for optimum fiber direction and layer thickness, using optimality criteria. Structural and Multidisciplinary Optimization, 36, 2, 2008, 159-167.

6. Soldatos K.P., T1marc1 T.: A unified formulation of laminated composite, shear deformable five-degreesof-freedom cylindrical shell theories. Composite Structures, 25, 1993, 165-171.

7. Timarci T., Soldatos K.P.: Comparative dynamic studies for symmetric cross-ply circular cylindrical shells on the basis of a unified shear deformable shell theory. Journal of Sound Vibration, 187, 4, 1995, 609-624.

8. Karaçam F.: MSc. Thesis (In Turkish), Trakya University, Institute of Science, Turkey, Edirne, 2005.

9. Goldberg D.E.: Genetic Algorithms in Search, Optimization and Machine Learning. Addison-Wesley Longman Publishing Co., Inc., USA, Boston, MA, 1989.

10. Karama K.S., Afaq S., Mistou S.: Mechanical behaviour of laminated composite beam by the new multi-layered laminated composite structures model with transverse shear stress continuity. International Journal of Solids and Structures, 40, 2003, 1525-1546. 